\title{
Fractional Sampling Improves Performance of UMTS Code Acquisition
}

\author{
Francesco Benedetto, Gaetano Giunta \\ Department of Applied Electronics, University of ROMA TRE, Rome, Italy \\ Email: fbenedet@uniroma3.it \\ Received March 31,2009; revised April 24,2009; accepted April 28, 2009
}

\begin{abstract}
An improved technique with a fractional sampling based on two samples per chip, according to the Nyquist criterion, has been employed by the authors to enhance the performance in the code synchronization of UMTS (or W-CDMA) systems. In this paper, we investigate on the theoretical rationale of such a promising behavior. The performance is analyzed for several wireless channels, in the presence of typical pedestrian and vehicular scenarios of the IMT2000/UMTS cellular systems.
\end{abstract}

Keywords: Code Synchronization, Spread Spectrum, Cell Search, UMTS, W-CDMA, Wireless Access

\section{Introduction}

Initial cell search in the wireless access of the International Mobile Telecommunications-2000/Universal Mobile Telecommunications System (IMT2000/UMTS) is the process of the mobile station that includes the search for cell and scrambling codes as far as time synchronization [1]. In fact, the whole synchronization process in Wideband-Code Division Multiple Access (W-CDMA) [2] consists of five sequential steps: 1) slot synchronization; 2) frame synchronization and scrambling code group identification; 3) scrambling code identification; 4) frequency acquisition; 5) cell identification. This contribution addresses algorithms for the initial cell search before frequency acquisition, i.e. the stages 1-3. The combined goal of such stages is to deliver a reliable code-time candidate to the frequency acquisition stage. Several sequential statistical tests were suggested for such purposes [3-9]. In particular, effective non-coherent sequential pseudo-noise (PN) code acquisition using sliding correlation were proposed and analyzed for both chip-asynchronous [7-8] and synchronous [9] direct sequence spread-spectrum (DS/SS) communications. According to such attempts, one testing variable is accumulated after correlation (sometimes implemented as the output of a matched filter) with each possible PN code shifted by each code offset. That is, the decision device sequentially examines all the code offsets of all the possible PN codes. The testing thresholds are optimally set to provide the probability of detection and false alarm, required for the considered application.

This paper extends previous analyses (e.g. [2]) that made the simplifying assumption of one sample per chip. Such choice appears motivated when a rectangular pulse waveform is employed for spread-spectrum modulation, since finer timing estimation can be confined to subsequent acquisition steps [3-4]. Nevertheless, the author of the papers [7-8] already discussed some improvement from over-sampling. Practical acquisition and signal processing requires more than one sample per chip. In fact, using only one sample could result in a significant performance loss [10]. With a more appropriate discretization, we are considering the effect of time-sampling the chip waveform (and the received signal) at twice the chip rate on the performance of detection and acquisition schemes. In fact, IMT2000/UMTS standard employs raised-cosine spectral waveforms with non-zero roll-off $[1,11]$, and it is then necessary to use receivers based on a fractional chip sampling, i.e. operating with more than one sample per chip.

The remainder of this paper is organized as follows. The use of fractional sampling to improve the performance of non-coherent sequential cell search procedure for PN code acquisition is presented and discussed in Sec- 
tion 2. The numerical results of the presented methods for application to the initial cell search (before frequency acquisition) of the IMT2000/UMTS cellular system is examined in Section 3 by a number of numerical results. Our conclusions are finally drawn in Section 4.

\section{Improving the Code Synchronization by Fractional Sampling}

In this paper, we match the conventional method, based on one sample per chip (like the operating cases reported in [2]), to the fractional technique operating at twice the chip rate. As already noted by [10], such choice matches the Nyquist criterion. Unfortunately, this choice doubles the computational complexity of receivers. Moreover, they must operate twice faster than a conventional receiver. Nevertheless, the current trend, providing higher and higher-speed microprocessors, is going to timely compensate for the augmented request of computational speed.

The author of the paper [7] already discussed some improvement from over-sampling. In particular, that original technique ("scheme 2" in [7]) implements the sampling of the received signal at twice the chip rate, but averages the samples at odd multiples of the half-chip period before a subsequent decimation by the factor 2 . As a result, the final data clock is always synchronous with the chip rate. In practice, the average operation of the "scheme 2" in [7] as well as the matched filter correlation by the "averaged" code $\bar{c}(t)$ in [8] are equivalent to using the filter with impulse response $h(t)=$ $\left[\delta\left(t-T_{\mathrm{c}} / 2\right)+\delta\left(t+T_{\mathrm{c}} / 2\right)\right] / 2$, corresponding to the low-pass frequency response $H(f)=\cos \left(\pi T_{c} f\right)$, before down-sampling, where $T_{c}$ is the chip period. In fact, it is well known [12] that some kind of anti-aliasing filtering is required before decimation to avoid the spectral alias error, at the cost of missing the information at frequencies higher than $1 / 2 T_{c}$ (while the spectrum of a raised cosine waveform is actually as large as $(1+R) / 2 T_{\mathrm{c}}$, where $R$ is the roll-off). Unlike the method based on 2 samples/chip proposed in this paper, the effect of the "scheme 2" in [7] is to filter out (or, at least, attenuate) the (significant) signal components in all the transition bandwidth [12], that is:

$$
\left[(1-R) / 2 T_{\mathrm{c}},(1+R) / 2 T_{\mathrm{c}}\right] \text {, because } H\left(1 / 2 T_{\mathrm{c}}\right)=0 \text {. }
$$

In accordance with the logic scheme [7], let us consider $D$ samples of the complex envelope of the received signal after the matched filter $\left\{r\left(i T_{\mathrm{c}}-\tau T_{\mathrm{c}}\right), i=1, \ldots, D\right\}$, discretized with the normalized timing offset $\tau$, being $\tau=0$ (without loss of generality) in the chip-synchronous case while (randomly distributed) $-0.5 \leq \tau \leq 0.5$ in chipasynchronous communications. The modified power detector operating at twice the chip rate first estimates the cross-correlation $\rho_{w}\left(k^{\prime} ; \tau\right)$ (despreading the generic $w$-th data block made of $D$ chips) between an over-sampled (by the factor 2) version of the received signal $r(t)$ after the matched filter and the code candidate $c(t)$ also for half-chip offsets $k T_{\mathrm{c}}$ :

$$
\begin{aligned}
\rho_{w}(k ; \tau) & =\frac{1}{2 D} \sum_{i=1}^{2 D} r\left(i T_{c} / 2-\tau T_{c}\right) \cdot c^{*}\left(i T_{c} / 2+k T_{c}\right) \\
\text { with } k & =-1.5,-1,-0.5,0,0.5,1,1.5
\end{aligned}
$$

As pointed out in [7], the correct timing offset is randomly located $(-0.5 \leq \tau \leq 0.5)$ in a chip-asynchronous system, being independent of the sampling times (either integer or half-integer multiples of the chip period $T_{\mathrm{c}}$ ). As pointed out in [6], the worst case of erroneous synchronization with the conventional power detector will happen if the cross-correlation is computed for an offset which is just in the middle between two chips. In such a case, an error of half chip will affect the estimates. In our method, the worst offset is located at $T_{\mathrm{c}} / 4$ instead of $T_{\mathrm{c}} / 2$. In practice, the maximum offset error is the half of the conventional detector. As a consequence, the worst case can be modeled by testing the null hypothesis $H_{0}$ against the worst positive hypothesis with $\tau=T_{\mathrm{c}} / 2$ (say $H_{0.5}$ ), for the conventional detector, and the worst positive hypothesis with $\tau=T_{\mathrm{c}} / 4$ (say $H_{0.75}$ ) for the devised over-sampled detector. In the conventional technique, the "middle" hypothesis lies at $\tau=T_{\mathrm{c}} / 4$ far from the correct timing offset $\tau=0$ (say $H_{0.75}$ ), while the "middle" positive hypothesis for the proposed approach is $\tau=T_{\mathrm{c}} / 8$ far from the correct offset $\tau=0$ (say $H_{0.875}$ ). It should be noted that the half-chip offset $\left(\tau=T_{\mathrm{c}} / 2\right)$ correlation could have a non-negligible impact on the acquisition performance. Nevertheless, in full agreement with the approach by Jia-Chin Lin [8], detecting the half-chip correlation sample $\left(H_{0.5}\right)$ is actually a metastable state that may sometimes lead to false-alarm conditions, but such conditions can be very easily corrected in the next test immediately. The mean acquisition time $A_{\mathrm{T}}$ of a serial search procedure over $q$ cells $(q>>1)$ is related to these probabilities according the approximate expression $[4-5]$ :

$$
A_{T} \cong q \cdot\left[L+P_{f a} T_{P}\right] \frac{2-P_{D}}{2 P_{D}}
$$

where $L=W D T_{\mathrm{c}}$ is the test's duration for each code offset candidate and $T_{p}$ is the penalty time for an erroneous acquisition while the signal does not actually exist. As a consequence, the ratio of the mean acquisition times, say $A_{\mathrm{T} 1}$ and $A_{\mathrm{T} 2}$, of two generic CFAR detectors with the same duration $L$, that is:

$$
\frac{A_{T 1}}{A_{T 2}} \cong \frac{\left(2-P_{D 1}\right) \cdot P_{D 2}}{P_{D 1} \cdot\left(2-P_{D 2}\right)}
$$


is able to directly evidence the approximate gain in saving time (on the average) of the latter against the former methods when the same signal data blocks are available.

In the following of this paper, we are going to compare the performance of the two methods with respect to the "middle" synchronization conditions, considered here as representative of the average operating conditions. The results of our computer simulations, conversely assuming a uniform distribution of the timing offset, are going to show that the "middle" case (defined for one given "middle" offset) is representative of such a random jitter of actual timing offsets.
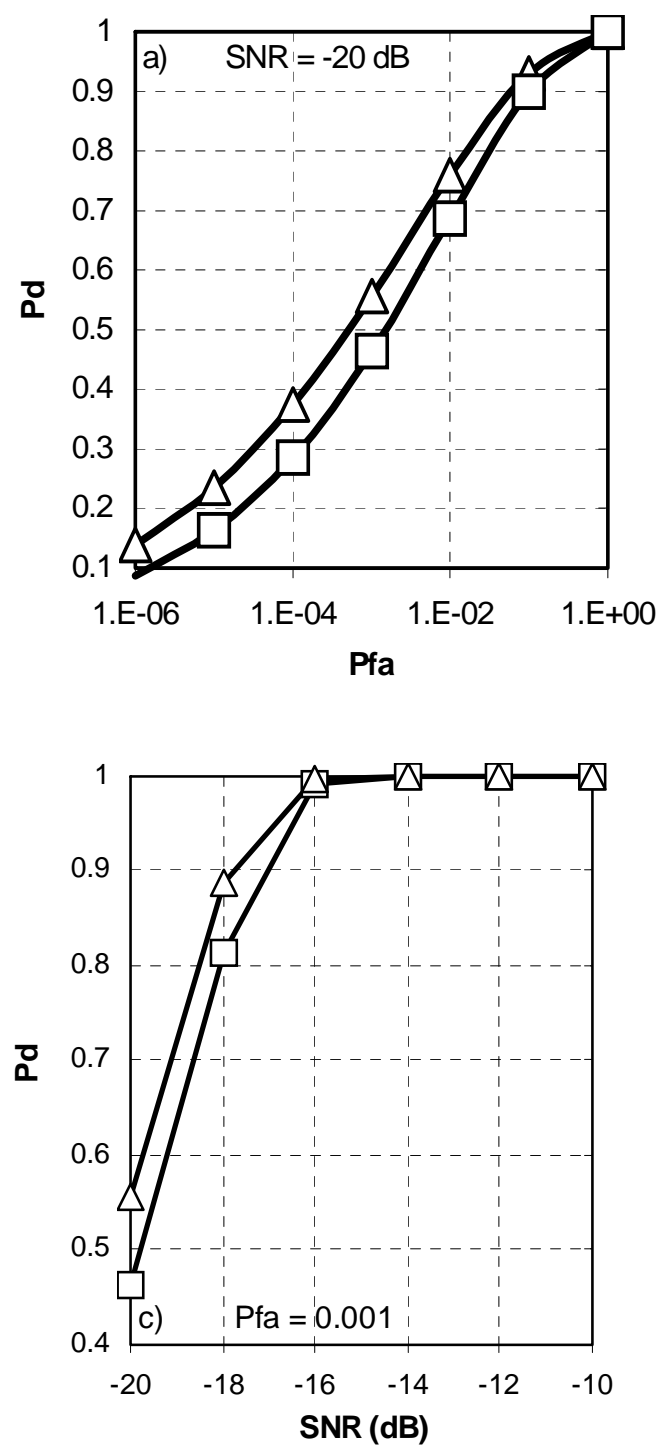

\section{Application to Initial Synchronization in UMTS and Results}

In this section, we aim to show that the analyzed methods for code synchronization are applicable to the first stages of initial cell search in the cellular UMTS system. In particular, we are going to evidence that the analytic expressions, reported in the previous sections, are able to predict the achievable gain on the error probability and the mean acquisition time of the over-sampled versus the conventional method. The same scenarios as in [2] of
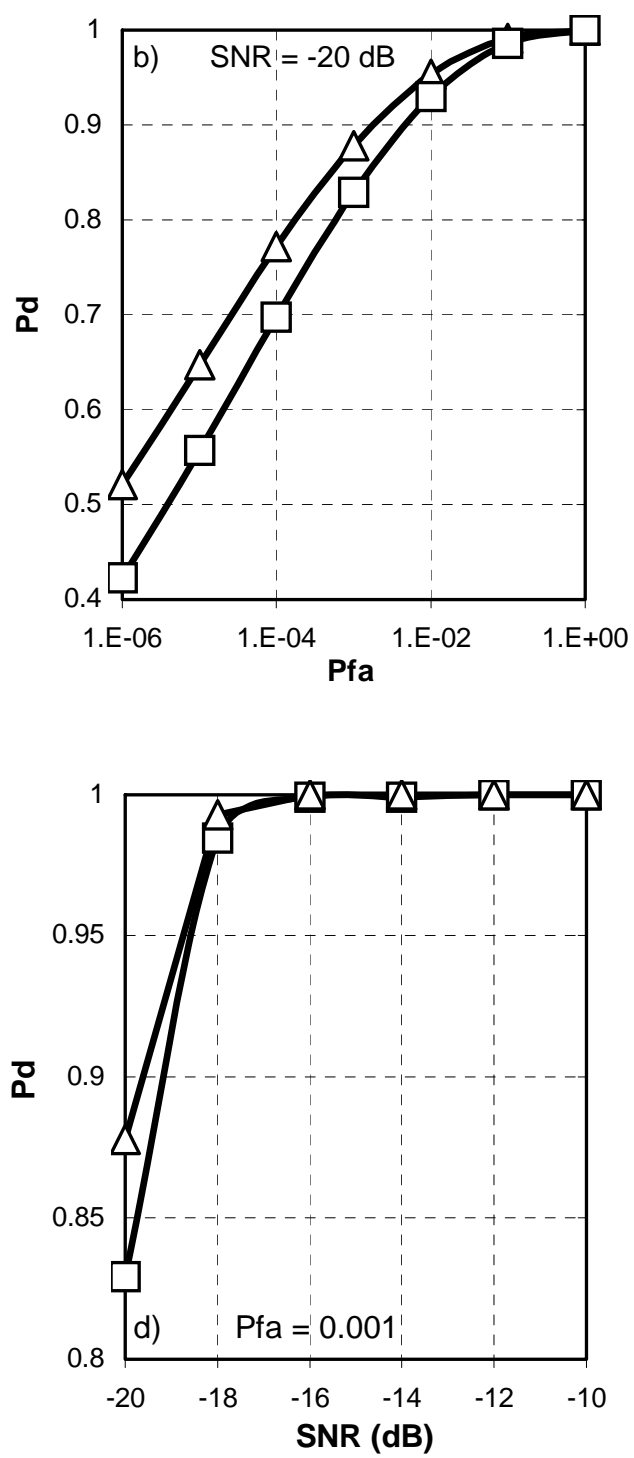

Figures 1a-d. Analytic probability of detection versus the probability of false alarm (top: a,b) and the SNR value per chip (bottom: c,d) in the "middle" offset case for a frequency uncertainty of $20 \mathrm{kHz}$ (left: a,c) and $200 \mathrm{~Hz}$ (right: b,d), matching the achieved performance of the conventional (squares) and over-sampled (triangles) methods for W=60 blocks of 64 chips (10 ms). 
cell search have been extensively studied. The frequency errors of $20 \mathrm{kHz}$ and $200 \mathrm{~Hz}$, typical of the initial and the target cell search, have been considered. In IMT2000/ UMTS [2], the pilot symbols available for code synchronization consist of 256 consecutive chips per slot (each slot is made of 2560 chips in total). The cross-correlation performance of one and more groups of frames (each made of 15 slots, i.e. $10 \mathrm{~ms}$ ), as far as the overall synchronization time in flat fading channels, has been analyzed. If one directly chooses $D=256$, the large frequency error during the initial search results in a large incoherence loss, especially in the initial search. This problem

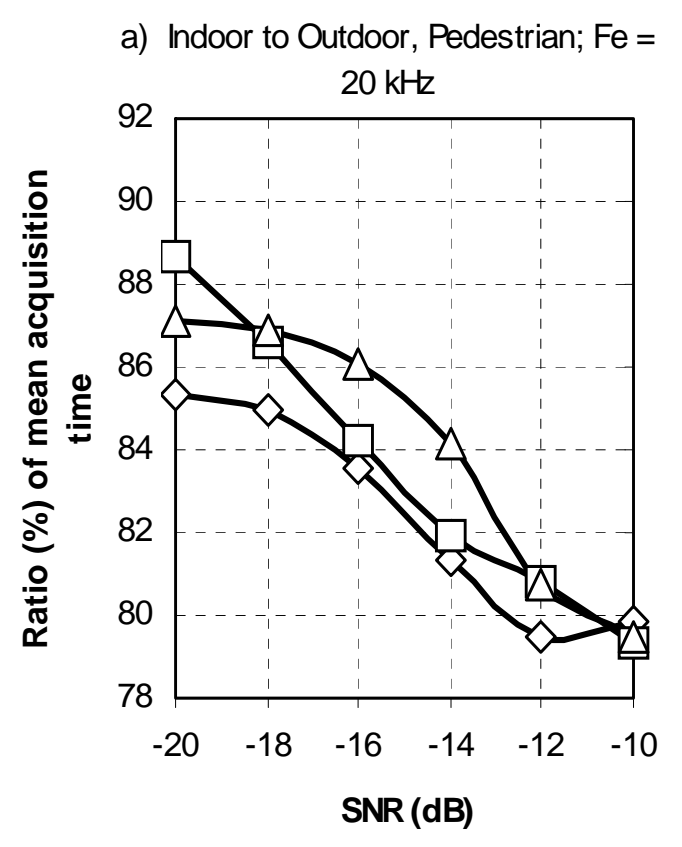

c) Vehicular; $\mathrm{Fe}=20 \mathrm{kHz}$

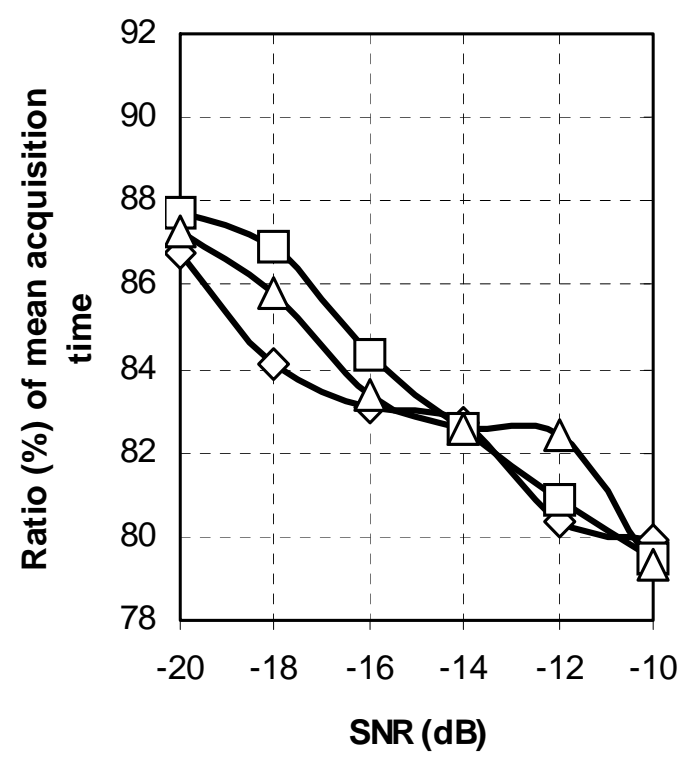

b) Indoor to Outdoor, Pedestrian; Fe = $200 \mathrm{~Hz}$

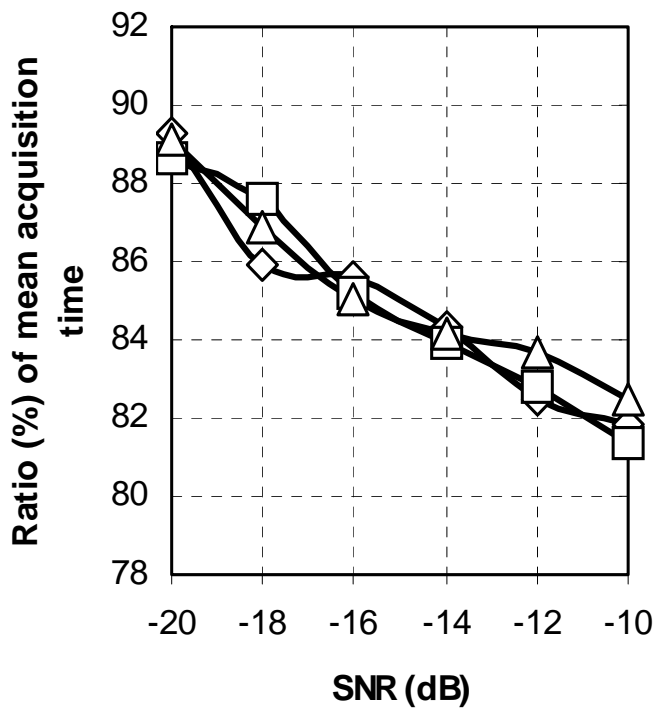

d) Vehicular; $\mathrm{Fe}=200 \mathrm{~Hz}$

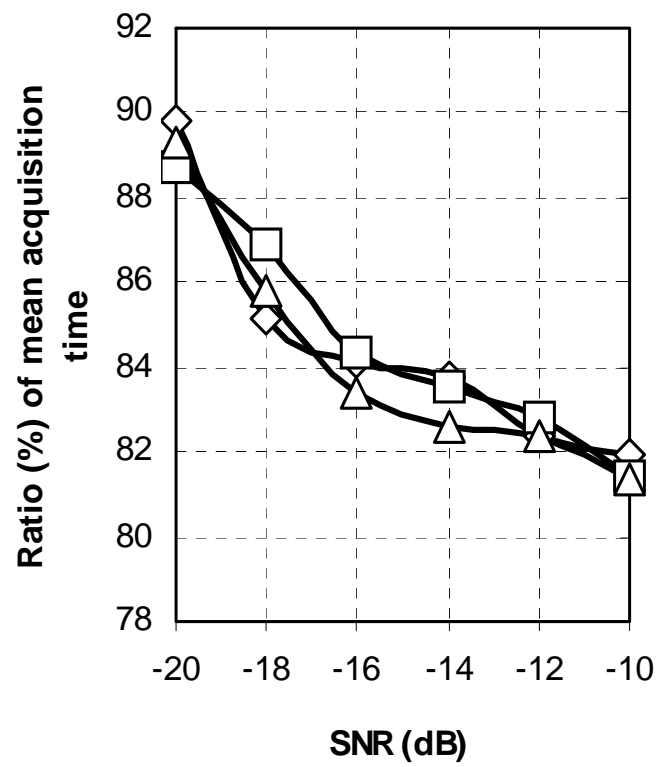

Figures 2a-d. Ratio of the experimental mean acquisition time of fractional and conventional procedures in the three initial stages (stage 1: diamonds; stage 2: squares; stage 3 triangles) versus the SNR per chip for pedestrian (top: a,b) and vehicular (bottom: c,d) scenarios with a random timing jitter for a frequency uncertainty of $20 \mathrm{kHz}$ (left: a,c) and $200 \mathrm{~Hz}$ (right: b,d). 
is solved by partial symbol despreading [2], using blocks of $D=64$ contiguous chips and non-coherent combining. In particular, the first stage of initial code synchronization provides a number of possible candidates of code offsets to the following one [2]. As a consequence, a sequential test can be implemented for such a purpose, performed like the procedure described in the former part of this paper. In the following (second and third) stages, only the most reliable code candidate is detected and finally processed by the frequency acquisition system [2].

The probability of detection in the "middle" synchronization case of the timing offset, defined in the previous section, is derived from the analytic expressions and depicted in figures $1 \mathrm{a}-\mathrm{d}$ versus the probability of falsealarm and the SNR for the two methods, in order to assess the validity of the over-sampled testing procedure in the presence of frequency uncertainties. The results of computer simulations, obtained with a uniformly distributed random jitter, have confirmed such trend. For sake of comparison, we have assumed the same typical pedestrian and vehicular scenarios reported in [2]. In particular, a uniformly distributed random timing jitter has been considered while the channel is affected by flat fading and three paths have been simulated, depending on the kind of scenario. Namely, pedestrian (speed: $3 \mathrm{Km} / \mathrm{h}$ ): the first path is $0 \mathrm{~dB}$ with delay $=0 \mathrm{~ns}$, the second one 0 $\mathrm{dB}$ and delay $=976 \mathrm{~ns}$, third one $0 \mathrm{~dB}$ and delay $=20000$ ns; vehicular (speed: $120 \mathrm{~km} / \mathrm{h}$ ): the main path is $0 \mathrm{~dB}$ with delay $0 \mathrm{~ns}$, the second one $-3 \mathrm{~dB}$ and delay $=260 \mathrm{~ns}$ and the third one is $-6 \mathrm{~dB}$ and delay $=521 \mathrm{~ns}$. Two possible constant frequency errors in the initial and target search [2] procedure (namely, $20 \mathrm{kHz}$ and $200 \mathrm{~Hz}$ ) are considered. In particular, the figures $2 \mathrm{a}-\mathrm{d}$ show the ratio of the mean acquisition time of the two methods versus the SNR per chip, obtained by Monte-Carlo simulations of the two reference wireless channels of [2] for $P_{f a}=0.001$, for the three initial stages of the serial code acquisition in UMTS.

Moreover, the same authors of this paper showed in [13], by extensive computer simulations, that the new scheme outperforms the conventional approach analyzing also the overall acquisition performance of the scrambling code, then including all the three steps of the initial synchronization procedure (before frequency acquisition). The authors, in [13], evidenced the significant real-time saving of the mean acquisition time (from 12\% to $21 \%$ ) of the suggested procedure, compared to the conventional technique, in the presence of multi-path channels with flat fading and frequency inaccuracy. In practice, the benefits on the mean acquisition time of using two samples per chip, since the first stage of code synchronization, are twofold: first, the cross-correlation between the received signal and the code's candidates are better estimated, then increasing the testing power of stage 1 (i.e. probability of correct detection for a constant false alarm rate); second, the timing error provided to the stages 2 and 3 is ideally the half of the conventional technique, being maximized by one fourth of the chip period.

\section{Conclusions}

This paper has addressed algorithms for initial code synchronization by sequential cell search, suited for application to the first three stages of the IMT2000/UMTS cellular wireless access system, i.e. initial cell search before frequency acquisition. The basic testing method, based on one sample per chip, has been herein matched to an improved technique based on a fractional chip sampling (and processing) that operates at twice the chip rate, according to the Nyquist criterion. The simulation analyses have evidenced a significant reduction of the mean acquisition time by the suggested procedure, compared to the conventional technique. In perspective, exploiting the half-chip offset correlation (by devising new well-performing testing variables) could further improve these schemes.

\section{Acknowledgments}

The authors wish to thank Prof. A. Neri and Dr. M. Carli of the University of Roma Tre for their support on the preliminary trials of numerical analysis and further discussions on application to 3G Mobile Systems.

\section{References}

[1] T. Ojanperä and R. Prasad, "An overview of air interface multiple access for IMT2000/UMTS," IEEE Communication Magazine, Vol. 36, pp. 82-95, September 1998.

[2] Y. Pin, E. Wang, and T. Ottosson, "Cell search in W-CDMA," IEEE Journal on Selected Areas in Communications, Vol. 18, No. 8, pp. 1470-1482, August 2000.

[3] R. L. Pickholtz, D. L. Schilling, and L. B. Milstein, "Theory of spread spectrum communications. A tutorial," IEEE Transactions on Communications, Vol. 30, pp. 855-884, May 1982.

[4] D. M. DiCarlo and C. L. Weber, "Multiple dwell serial search: Performance and application to direct-sequence code acquisition," IEEE Transactions on Communications, Vol. 31, pp. 650-659, May 1983.

[5] G. Giunta, "Generalized Q-Functions for application to non-coherent serial detection of spread-spectrum communication signals," IEEE Transactions on Signal Processing, Vol. 48, No. 5, pp. 1506-1513, May 2000.

[6] J.-C. Lin and C.-Y. Lin, "Non-coherent sequential PN code acquisition using sliding correlation in DS/SS," In- 
ternational Conference on Communication, ICC'2000, pp. 341-345, 2000.

[7] J.-C. Lin, "Noncoherent sequential PN code acquisition using sliding correlation for chip-asynchronous direct-sequence spread-spectrum communications," IEEE Transactions on Communications, Vol. 50, No. 4, pp. 664-676, April 2002.

[8] J.-C. Lin, "Differentially coherent PN code acquisition based on a matched filter for chip-asynchronous DS/SS communications", IEEE Transactions on Vehicular Technology, Vol. 51, No. 6, pp. 1596-1599, November 2002.

[9] J.-C. Lin, "Differentially coherent PN code acquisition with full-period correlation in chip-synchronous DS/SS receivers," IEEE Transactions on Communications, Vol. 50, No. 5, pp. 698-702, May 2002.

[10] A. Mantravadi and V. V. Veeravalli, "On chip-matched filtering and discrete sufficient statistics for asynchronous band-limited CDMA systems," IEEE Transactions on
Communications, Vol. 49, No. 8, pp. 1457-1467, August 2001.

[11] E. Dahlman, P. Beming, J. Knutsson, F. Ovesjo, M. Persson, and C. Roobol, "WCDMA-The radio interface for future mobile multimedia communications," IEEE Transactions on Vehicular Technology, Vol. 47, pp. 1105-1118, November 1998.

[12] W.-H. Sheen, J.-K. Tzeng, and C.-K. Tzou, "Effects of cell correlations in a matched filter PN code acquisition for direct-sequence spread spectrum systems," IEEE Transactions on Vehicular Technology, Vol. 48, No. 3, pp. 724-732, May 1999.

[13] F. Benedetto, M. Carli, G. Giunta, and A. Neri, "Performance benefits of fractional sampling in the initial code synchronization for the wireless access of $3 \mathrm{G}$ mobile communications," IEEE International Vehicle Technology Conference, VTC 2005-Spring, Stockholm, Sweden, June 2005 